\title{
Modeling of Ferro-resonance on the VT of GIS substation
}

\author{
S.M.Hassan.Hosseini ${ }^{1}$, Y. Toghani Holari ${ }^{2}$, S.M.Hosseini.Bafghi ${ }^{3}$ \\ 1, 2, 3 (Department of Electrical Engineering, Islamic Azad University South Tehran Branch, Tehran, IRAN)
}

\begin{abstract}
The main subject of the essay is study of ferroresonance in the voltage transformers at $400 \mathrm{kV}$ GIS substation. Ferroresonance has destructive effects such as, undulations of high voltages, warming of transformers, increasing of heat, temperature increasing and damaging voltage transformers, high noise due to evolution due to magnet and bad working of protective instruments. So we have to apply limitations on system parameters to avoid such an undesirable phenomenon like this. In this paper ferroresonance phenomenon on VTs of real GIS substation (karon 4 power plant had been studied. With regarding high risk of ferroresonance due to studied substation conditions, damping circuit (FDSD, ZD) had been designed with help of EMTP-RV and the results are presented in two conditions of being without damping and by using of damping in the circuit. The results show damping circuit is successful in obviation of these dangerous oscillations.
\end{abstract}

Keywords: Gas Insulated Substation (GIS), Voltage Transformers (VT), Ferroresonance, FDSD, ZD

\section{INTRODUCTION}

Regarding to the advantages of GIS substations in comparison with AIS (Air Insulated Substation) and purposing that instruments such as current and voltage transformer, bypass disconnector switch, high voltage conductor, grounding switchgear, breaker and etc. that are kept in boxes under pressure of SF6 gas, and increasing usage of these substations toward the past and regarding to this point that urban and industries areas with increasing load and insufficient area and needing high voltages are regarded, in last year's the use of GIS substation have increased.[1]. In this paper we study the Karoon 4 power plant GIS substation that is a $400 \mathrm{kV}$ substation with double bus bar and disconnector switch, this substation is fed by 4 single phase 100MW transformers. The outputs of these transformers are connected to the $400 \mathrm{kV}$ network with 4 air lines. Despite the advantages of this gas insulated substations, it has fundamental problems. One of the main ingredients of destruction of the instruments in GIS substations is ferroresonance that influenced the insulation of network at different point. Ferroresonance is one of the rare and destructive phenomena in power network that figured as a nonlinear resonance that happen between the capacitor of the network and the nonlinear inductance of voltage transformer at the saturation instant. The word of ferroresonance, that arrived to the books and papers from the end of the second decade of 20th century, use for all the oscillation phenomena in electrical circuits that consist of at least nonlinear self, capacitor, voltage source (generally sinusoidal). The effective elements in causing resonance and ferroresonance in power systems are, harmonic, core saturating, changing inductance of wiring, cutting of 1 or 2 phases of system, unsymmetrical load, inappropriate use of circuit breakers and fuses, using cables with high capacitance. Ferroresonance has other destructive effects such as oscillation of high voltages, intricacy in wave forms of voltage and current, warming of transformers, damaging voltage transformers (VT), for example in Karoon 4 power plant 400kV GIS substation, loud noise due of evolution due to magnet, malfunction protective instruments. Numerous cases of ferroresonance in power system have been reported in years. In last year's the number of ferroresonance because of the intricacy of system and operating modern instrument has increased. [2], [3] outbreak of ferroresonanc has some signs like permanent over voltage with vast amplitude as phase to phase or phase to ground, permanent over current with vast amplitude, shifting voltage zero point, increasing temperature of voltage transformer, continual increasing of the noise altitude of transformers and reactors, damaging electrical equipment's such as capacitor banks and CVT because of increasing the thermal effect or breakage of dampers of the protective equipment's. Therefore some limitations should be applied to parameters of systems to avoid occurrence of undesirable phenomena like these. [4], [5]. All of the studies have been simulated by EMTP-RV software.

\section{Parallel Capacitor In H.V Circuit Breaker}

Power capacitors have different usage in structure and mechanism of power circuit breaker especially at high voltage levels. Like grading capacitors that have two breaking boxes in series. Voltage usually doesn't distribute equally on breaking boxes. To avoid making stress of high voltage and smoothing voltage on them, capacitors parallel with contacts of circuit breaker that usually have capacity between 900 to $1600 \mathrm{pF}$ for each breaking box are used. Although by the development of technology usage of these capacitors have been reduced but they are used in high voltages yet.[3],[6]. Some defects of these capacitors are increasing the risk of 
ferroresonance with nearby voltage transformers, increasing the weight, complexity of installation operation and increasing leakage current.

\section{Karoon 4 Case Study}

The purpose of this paper is to study the ferroresonance on voltage transformers (VT) in Karoon 4 power plant GIS 400kv substation as an example of GIS substation with bus bar and long line that have been effected by this phenomenon by increasing the voltage and damaging isolation and heating, destruction and damaging of VT . These simulations are done by EMTP-RV Software and finally propose the practical method to resist it. There conductive wires of $\mathrm{A} \& \mathrm{C}$ phase of damping reactor have been burnt by the high current at secondary circuit because of the single phase with VT ferroresonance. One of the reasons of this phenomenon is that the VT Company adequately doesn't consider the capacity of the damping reactor to absorb the resonance energy in VT in some cases of GIS switching conditions during first energizing at site. [7] Special recommendation presented in this paper offer to avoiding over heating Beside VT of BUS 1 and 2 and damping reactor until additional FDSD (Ferroresonance Detecting and Suppression Device) at site became adjusted. The related circuit breaker (CB) for each $400 \mathrm{kV}$ GIS bay should be closed about 5 minutes after closing of both DS's (disconnecting switches) during energization. Reversely, during power Outage one of both DS's should be opened within 5 minutes after opening of the circuit breaker [3], [8]. On $19 \& 24$ November in 2010, VT of \#1 bus was exposed to ferro-resonance 2 times during energizing (10 min.) \& de-energizing ( $2 \mathrm{~min}$.) by switching of CB \& DS of C03 bay that is shown is shown in Figure 1.

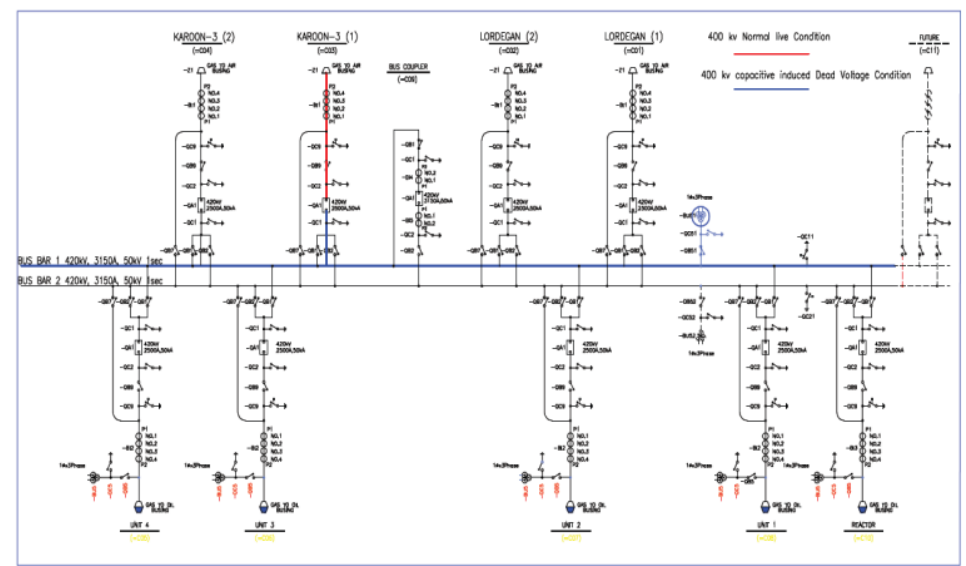

Fig1. Karoon4 400kV substation single line diagram state of energizing from first circuit output line of Karon3 and bus 1 and corresponding VT

On $20 \& 21$ November in 2010, VT of \#2 bus were exposed to ferroresonance 2 times during energizing (5min.) and de-energizing (2min.) by switching of CB \& DS of C04 bay through \#2 bus bar.

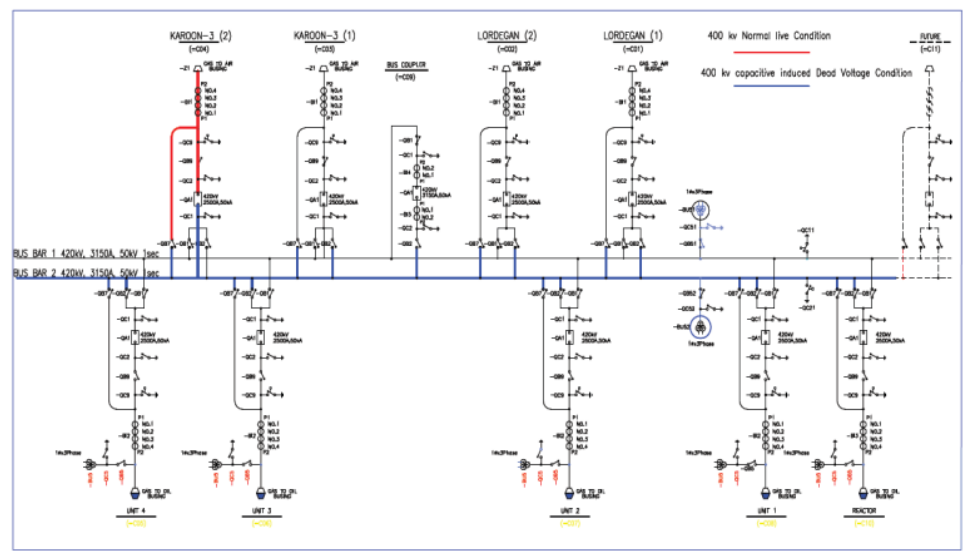

Fig2. Karoon4 400kV substation single line diagram state of energizing from second circuit output line of Karoon 3 and bus 2 and corresponding VT 


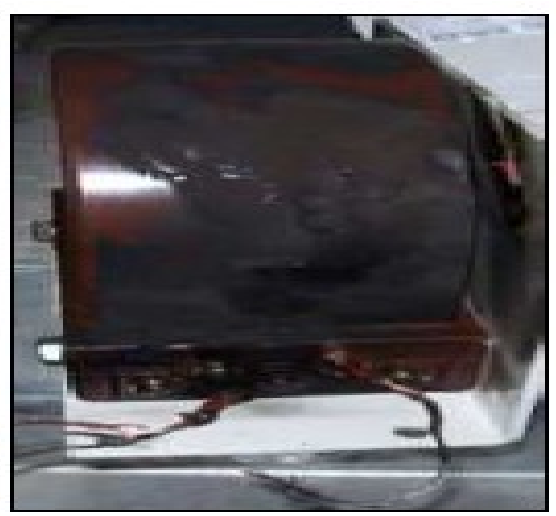

Fig3.Burnt Damping Reactor (ZD) of A-phase for \#2 Bus VT

\section{Ferroresonance Detection Supervision Devise (Fdsd)}

By regarding presented figures of this part such as fig 4 , a VT with conversion rate of $400 / \sqrt{T}$ to $110 / \sqrt{T}$ connected to a ferroresonace detector circuit is shown .[2].

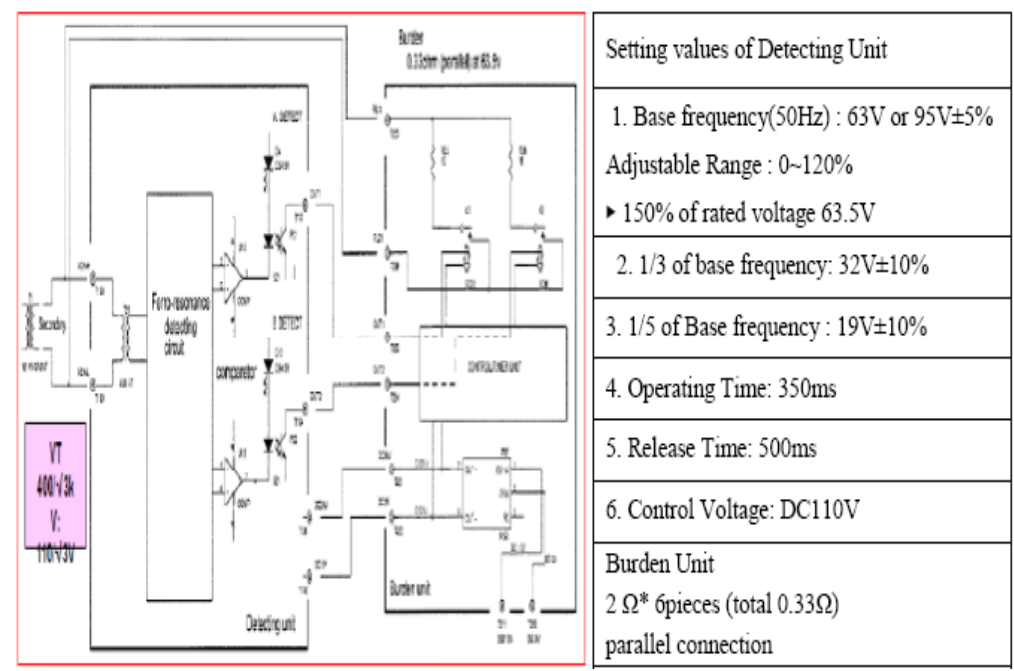

Fig4. Information for adjusting FDSD

In continue internal circuit of detecting unit is connected to burden with value of 0.33 that is equivalent of parallel branches. and also in figures 5 and 6, appearance of a FDSD and different part of operating lamp A,B , Power Lamp, sensitivity Change, Test Switch, Optional Output Control in three phase and single phase detector unit is shown.
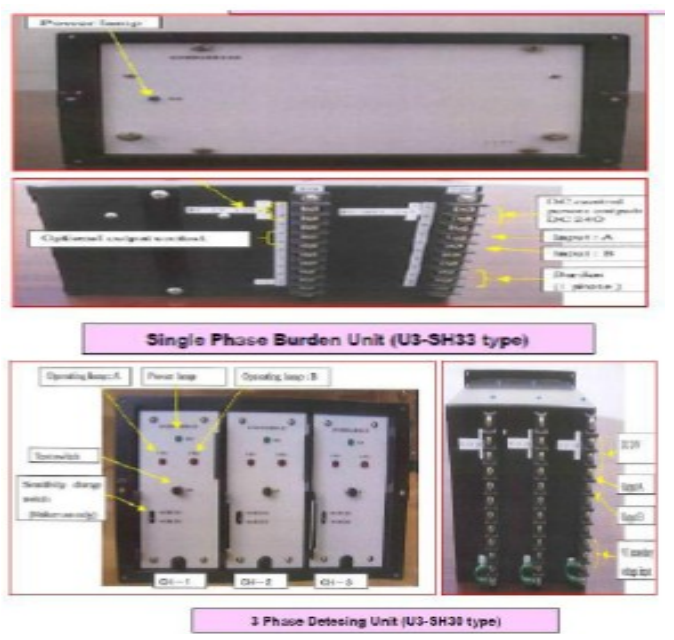

Fig5 .picture of seeming figure of FDSD 


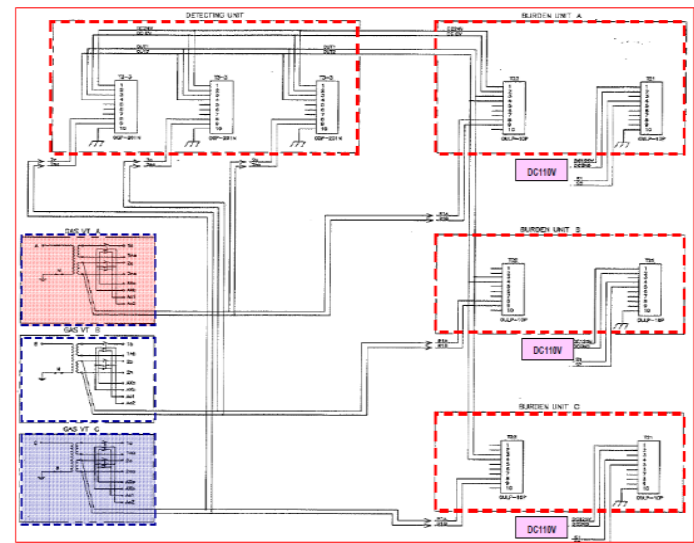

Fig6. Diagram of three phase FDSD connection

V. Ferroresonance Analysis With Fdsd $+Z$ Zd

The conditions of standard analysis that can be used in done simulation are as below:

1. CB Capacitance $=4,000 \mathrm{pF} / \mathrm{phase}$,

2. Unit Earth Capacitance for GIS $=52.4 \mathrm{pF} / \mathrm{m}$

3. Internal Earth Capacitance of M.Tr: 13,366pF/phase

4. Internal Earth Capacitance of in Shunt Reactor: 3,382pf/phase

In above analysis, karoon 4 power plant GIS substation, that is a $400 \mathrm{kV}$ substation with double bus bar with bypass disconnector switch, is studied and equivalent circuit of GIS substation, that consist of main parameters like, source and resistance series with it, and CB that capacitor $\mathrm{C} 1$ is parallel with it, and as explained at section 3 above GIS circuit breaker had two 2000pF capacitor parallel with contacts of circuit breaker and equivalent capacitance is 4000pf. The simulation is done on the basis of these values. Above simulated circuit consist of equivalent capacitor of GIS substation in different studied conditions that are as a result of summation of existing parameters which is studied in bellow waveforms. Also in this circuit, one VT with prime voltage of $400 \mathrm{kV}$ and secondary voltage of $105 \mathrm{~V}$ is used for simulation and at last ZD and FDSD equivalent circuit for damping probability of ferroresonance due to 4000pf capacitor parallel with CB are put .[7],[9].

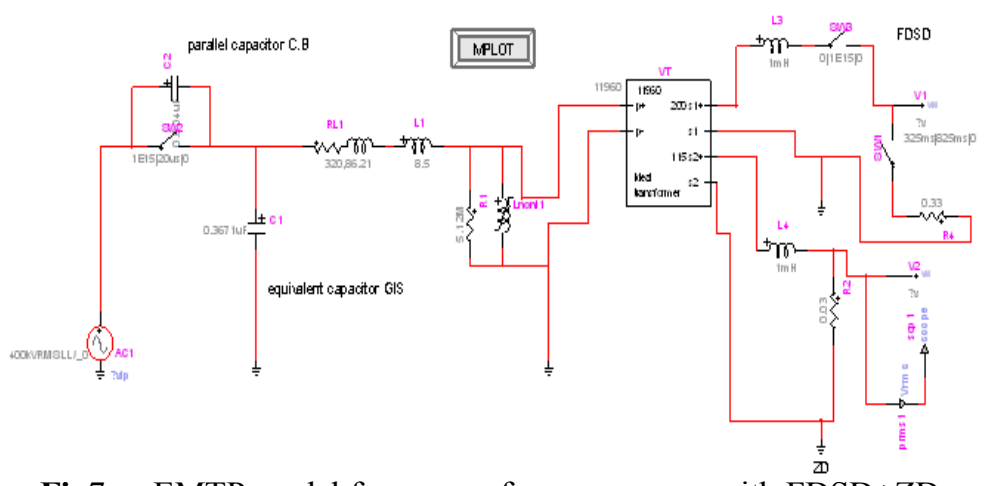

Fig7-a- EMTP model for survey ferroresonance with FDSD+ZD

- if $\mathrm{C} 1=4000 \mathrm{pF}$ and $\mathrm{C} 2=3820 \mathrm{pF}$ (for VT of bus 1 )

Which capacitor $(\mathrm{C} 2=(53.2+5.6+5.6+2+6.5) * 52.4=3820)$.

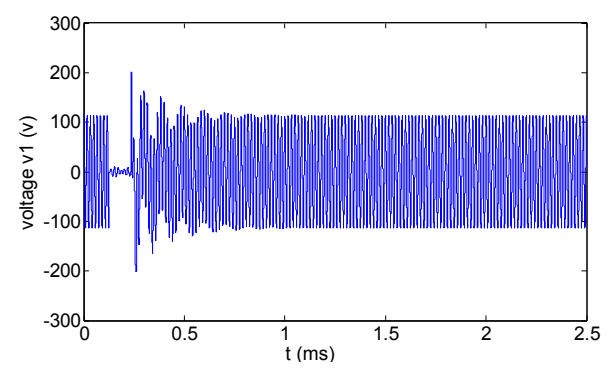

Fig7-b- voltage waveform of VT secondary voltage in damping ferroresonance (V1) 


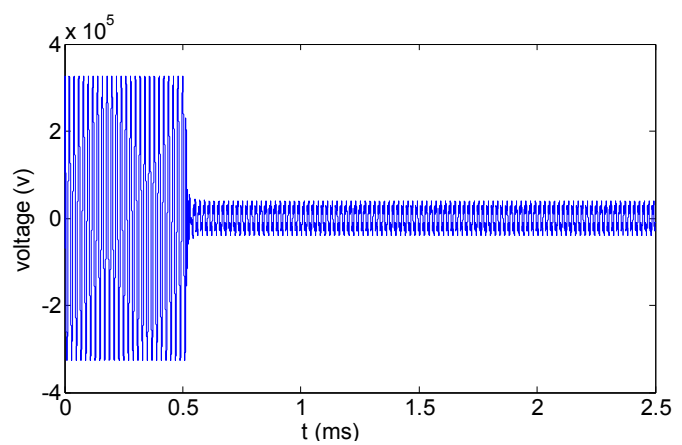

Fig7-c- voltage waveform of VT primary

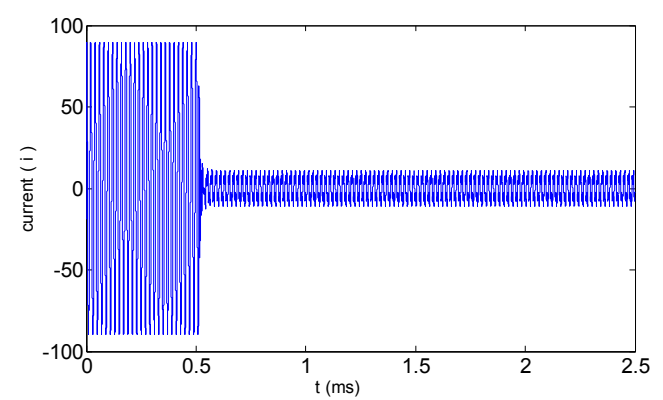

Fig7-d- current waveform of VT primary

- if $\mathrm{C} 1=4000 \mathrm{pf}$ and $\mathrm{C} 2=7669 \mathrm{pf}$ ( for VT bus )

Which capacitor $(\mathrm{C} 2=(53.2+5.6+7.05+7.2 * 9+2+7.2+6.5) * 52.4=7669)$

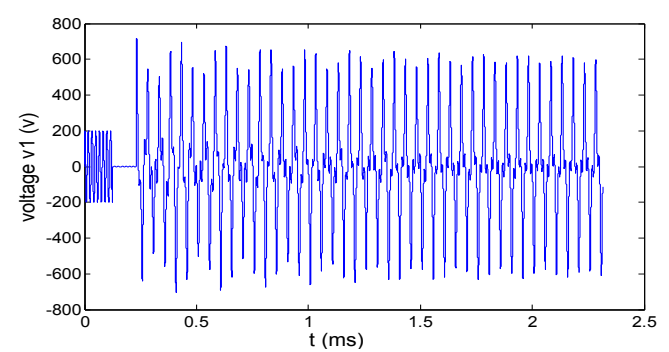

Fig8-a- VT secondary voltage waveform in presence of ferroresonance (V1)

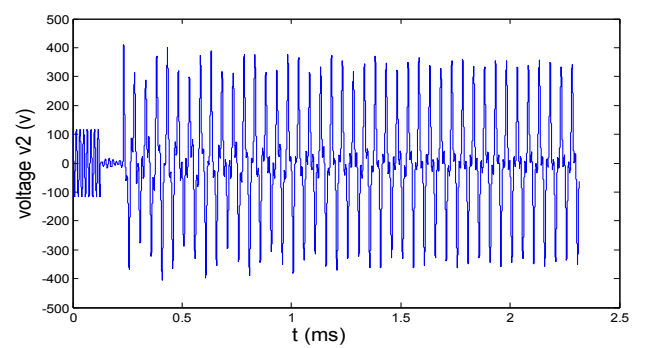

Fig8-b- VT secondary voltage waveform in presence of ferroresonance (V2)

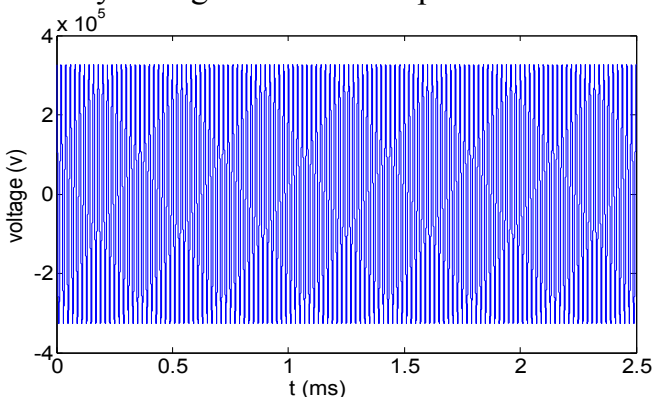

Fig8-c- used voltage source waveform in simulated diagram with EMTP 


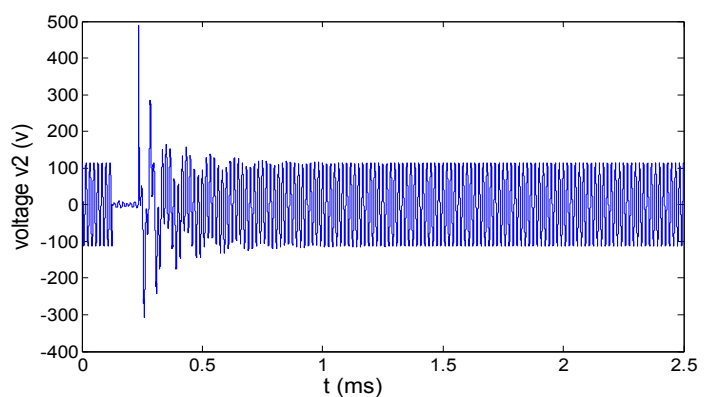

Fig8-d- VT secondary voltage waveform in damping ferroresonance (V2)

The structure of gas switchers generally act like a capacitor (energized conductor-gas insulatorgrounded body). Also because of the mentioned reasons in high voltages, capacitors parallel with breaker contacts are used. Capacitor parallel with breaker contacts are in series with GIS switcher capacitor, therefore always there is a path for transmission of capacitor current, even when the breaker is in open state. This current cause the voltage division between two capacitors and whatever the ratio of breaker parallel capacitor to GIS capacitor is larger (lower impedance), voltage after circuit breaker and on the bus bar will be larger. GIS circuit breaker existing in mentioned substation has two 2000pf capacitors parallel with breaker contact that the equivalent capacitance will be 4000pf and can cause the ferroresonance on equipment's like VT. Different producers of gas switchers use these capacitors to increase the operation of their circuit breakers. [8], [9]. One effect of ferroresonance due to breaker parallel capacitor is heating and burning VT in Karoon 4 power plant $400 \mathrm{kV}$ GIS substation that is one of problems in such substation that their equipment are made by Hyundai company and other several company.

\section{CONCLUSION}

In this paper ferroresonance phenomenon on voltage measuring transformer in real $400 \mathrm{kV}$ GIS substation (karon4 power plant) has been studied. After simulation, fig 7-b and 7-c show primary and secondary voltage waveforms of VT and fig 7-d shows primary current of VT for bus number 1. Fig 8-a and 8-b show secondary voltage waveform of VT in presence of ferroresonance for bus 1 and bus 2 in sequence. Fig 8-c shows the source used in simulated diagram with EMTP and fig 8-d shows the secondary voltage waveform of VT in damping ferroresonance for bus 2. The corresponding model has been presented and for different conditions, simulation proved ferroresonance on VTs of bus 1 and bus 2. To eliminate this phenomenon, ZD+FDSD damper circuit had been designed and added to model, results of simulation showed success damping of these ferroresonance oscillations.

\section{REFERENCES}

[1] K. H. Tseng P.-Y. Cheng Department of Electrical Engineering, National Taipei University of Technology, No. 1, Sec. 3, ChungHsiao E. Rd.,"Mitigating $161 \mathrm{kV}$ electromagnetic potential transformers' ferroresonance with damping reactors in gas-insulated switchgear"Published in IET Generation, Transmission \& Distribution Received on 27th June 2010.

[2] Hyundai Reports "ferroresonance of VT "Hyundai Heavy Industries Co., LTD ,Electro Electric System Div./Electro-Mechanical Research Institute 2011.

[3] David A. N. Jacobson, Member IEEE "Examples of Ferroresonance in a High Voltage Power System "2003 IEEE.

[4] Q ing Liu, Yufeng Zhang "Influence of Switching Conditions on Very Fast Transient Over-voltage in500kV Gas Insulated Substation" Department of Electrical and Control Engineering Of Xi' an University of Science and Technology, Xi'an, 710054, China.

[5] SaeedShahabi, ElectricalEngineering Department, Iran University of Science \& Technology, Rasul EsmaeilniaShirvani, Islamic Azad University of Saveh, Mohammad Purrezagholi, Babol University of Technology "Mitigating Ferroresonance in Coupling Capacitor Voltage Transformers with Ferroresonance Suppressing Circuits"C2009 IEEE.

[6] H. Mohseni, J. Jadidian, A. A. Shayegani-Akmal, E. Hashemi, A. Naieny and E. Agheb "In-situ Insulation Test of 400 kV GIS", IEEE Transactions on Dielectrics and ElectricalInsulation Vol. 15, No. 5; October 2008.

[7] D. Shoup, Member, IEEE, J. Paserba, Fellow, IEEE, D. Sullivan, Member, IEEE P. Bolin, Senior Member, IEEE, R. Whiteside, Seni or Member, IEEE "ferroresonance Analysis of 500 kv Gas-Insulated substation "2011 IEEE.

[8] A. Abbasi, and A. Seifi, "Fast and perfect damping circuit for ferroresonance phenomena in coupling capacitor voltage transformers," Journal of Electric Power Components and Systems, Apr. 2009.

[9] W. Piasecki, M. Stosur, M. Florkowski, M. Fulczyk, B. Lewandowski"Mitigating Ferroresonance in HV inductive", Presented at the International Conference on Power System Transients (IPST'09) in Kyoto, Japan on June 3 -6, 2009. 